BNL-112538-2016-JA

\title{
Ground State of Underdoped Cuprates in Vicinity of Superconductor-to-Insulator Transition
}

\author{
Jie Wu, Anthony T. Bollinger, Yujie Sun, Ivan Božović
}

Submitted to Journal of Superconductivity and Novel Magnetism.

August 2016

\author{
Condensed Matter Physics and Material Science Department \\ Brookhaven National Laboratory
}

\author{
U.S. Department of Energy \\ USDOE Office of Science (SC), \\ Basic Energy Sciences (BES) (SC-22)
}

Notice: This manuscript has been authored by employees of Brookhaven Science Associates, LLC under Contract No. DE-SC0012704 with the U.S. Department of Energy. The publisher by accepting the manuscript for publication acknowledges that the United States Government retains a non-exclusive, paid-up, irrevocable, world-wide license to publish or reproduce the published form of this manuscript, or allow others to do so, for United States Government purposes. 


\section{DISCLAIMER}

This report was prepared as an account of work sponsored by an agency of the United States Government. Neither the United States Government nor any agency thereof, nor any of their employees, nor any of their contractors, subcontractors, or their employees, makes any warranty, express or implied, or assumes any legal liability or responsibility for the accuracy, completeness, or any third party's use or the results of such use of any information, apparatus, product, or process disclosed, or represents that its use would not infringe privately owned rights. Reference herein to any specific commercial product, process, or service by trade name, trademark, manufacturer, or otherwise, does not necessarily constitute or imply its endorsement, recommendation, or favoring by the United States Government or any agency thereof or its contractors or subcontractors. The views and opinions of authors expressed herein do not necessarily state or reflect those of the United States Government or any agency thereof. 


\section{Ground State of Underdoped Cuprates in Vicinity of Superconductor-to-Insulator Transition}

Jie Wu ${ }^{1}$, Anthony T. Bollinger ${ }^{1}$, Yujie Sun ${ }^{1,2}$, Ivan Božović ${ }^{1,3, *}$

${ }^{I}$ Condensed Matter Physics and Materials Science Division, Brookhaven National Laboratory, Upton, New York 11973-5000, USA

${ }^{2}$ Institute of Physics, Chinese Academy of Sciences, Beijing 100190, China

${ }^{3}$ Applied Physics Department, Yale University, New Haven CT 06520, USA

* To whom correspondence should be addressed. Tel: (+1)631-344-4973. Fax: (+1)631-3444071.Email:bozovic@bnl.gov 


\begin{abstract}
When an insulating underdoped cuprate is doped beyond a critical concentration $x_{c}$, hightemperature superconductivity emerges. We have synthesized in a series of $\mathrm{La}_{2-\mathrm{x}} \mathrm{Sr}_{\mathrm{x}} \mathrm{CuO}_{4}$ (LSCO) samples using the combinatorial-spread technique that allows us to traverse the superconductor-to-insulator transition (SIT) in extremely fine doping steps, $\Delta x \approx 0.00008$. We have measured the Hall resistivity $\left(\rho_{H}\right)$ as a function of temperature down to $300 \mathrm{mK}$ in magnetic fields up to $9 \mathrm{~T}$, At very low temperatures, $\rho_{H}$ shows erratic behavior, jumps and fluctuations exceeding $100 \%$, hysteresis, and memory effects, indicating that the insulating ground state is a charge cluster glass (CCG). Based on the phase diagram depicted in our experiment, we propose a unified picture to account for the anomalous electric transport in the vicinity of the SIT, suggesting that the CCG is in fact a disordered and glassy version of the charge density wave.
\end{abstract}

Keywords: high temperature superconductors | charge glass | superconductor-to-insulator transition | quantum fluctuations | Hall effect 
Copper-oxide superconductors undergo a superconductor-to-insulator transition (SIT) at a critical doping level $\left(x_{c} \approx 0.06\right)$. An important question here is what is the nature of the insulating state from which superconductivity emerges, since this is the environment within which Cooper pairs emerge and establish phase coherence. The undoped 'parent' compound $\mathrm{La}_{2} \mathrm{CuO}_{4}$ (LCO) is generally considered to be a Mott insulator ${ }^{1}$. However, behavior of underdoped $\mathrm{La}_{2-\mathrm{x}} \mathrm{Sr}_{\mathrm{x}} \mathrm{CuO}_{4}$ (LSCO) with $x \approx x_{c}$ deviates remarkably from that of a typical insulator. Although its resistivity $(\rho)$ increases as the temperature $(T)$ is reduced, its magnitude stays at or below the $\mathrm{m} \Omega \mathrm{cm}$ level, which is typical of a bad metal rather than of an insulator. In more detail, $\rho(T)$ shows an unusual $\log (1 / T)$ dependence and then saturates at a finite value $\mathrm{v}^{2-4}$ as $T \rightarrow 0$, in contrast to the behavior of a common insulator with exponential $\rho(T)$ dependence and $\rho(T)$ diverging as $T \rightarrow 0$.

The above facts raise the question whether a simple picture of holes hopping within a Mott insulator is sufficient to account for the experimental observations. Growing experimental evidence indicates that the picture is more complex, involving various forms of spin and charge ordering and localization. Muon spin reflectivity $(\mu \mathrm{SR})$ experiments ${ }^{5-7}$ revealed that when the temperature is lowered and the thermal fluctuations calmed down, in underdoped cuprates the spins of carriers form static patterns with random orientations - a spin glass state. Note that this state does not arise from the magnetic dipole-dipole interaction, which is several orders of magnitude weaker than the energy scale required to freeze spins. Rather, the spin and charge degrees of freedom are strongly coupled through the spin-orbit interaction. In this case, the glassy distribution of spins may be related to its counterpart, a glassy distribution of charges or the 'charge-cluster glass' (CCG). Indeed, magneto-transport experiments have detected hysteretic behavior of magneto-resistance, which was attributed to formation of the CCG state $e^{8-11}$. Here, we explore the validity of this scenario by systematically investigating the doping-driven SIT in LSCO. We tune the Sr doping level x in unprecedentedly fine steps, $\Delta x \approx 0.00008$, and use the Hall resistivity $\left(\rho_{H}\right)$, instead of magnetoresistance as the probe, greatly enhancing the sensitivity.

For this study, we have synthesized a very high quality, single-crystal LSCO thin film using atomic-layer-by-layer molecular beam epitaxy (ALL-MBE). The atomic sources were positioned at a shallow angle $\left(20^{\circ}\right)$ with respect to the surface of the $\mathrm{LaSrAlO}_{4}$ (LSAO) 
substrate. In this way, we ensure a constant gradient of the deposition rate across the substrate, which drops linearly from one edge of the substrate to the other; the total variation is $4 \%$ over the distance of $10 \mathrm{~mm}$. In consequence, the concentration of $\mathrm{Sr}$ dopants $x$ varies across the film at the same rate.

By photolithography, the film was etched into a Hall bar oriented in the direction of maximal gradient. To allow for position-dependent measurements of longitudinal and Hall resistivity, 64 gold contacts were attached at different positions to the Hall bar, this defining 32 'pixels', each with a different average doping level. Note that the difference in $x$ between two neighboring pixels is miniscule, $\Delta x \approx 0.00008$.

The transport measurements were performed using a He3 cryostat equipped with a superconducting magnet. A home-built multi-channel lock-in setup allows data collection from 32 channels simultaneously; we can measure both $\rho$ and $\rho_{H}$ at once for 30 different doping levels, at temperatures down to $300 \mathrm{mK}$ and in magnetic fields up to $9 \mathrm{~T}$. The details of this methodology have been described elsewhere ${ }^{12-14}$.

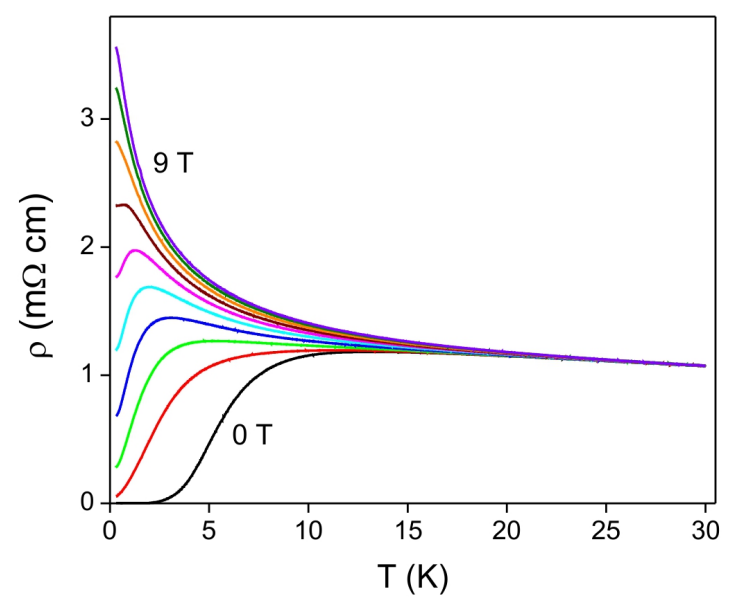

Fig. 1. The temperature dependence of the longitudinal resistivity $\rho(T)$ in magnetic field varied from $B=0$ to $B=9 \mathrm{~T}$ in steps of $1 \mathrm{~T}$, for one representative doping, $x$ $=0.062$. In zero field, $\rho(T)$ shows a superconducting transition with $T_{c} \approx 2 \mathrm{~K}$ (black curve). Magnetic field suppresses superconductivity and drives it towards an 'insulating' state characterized by the negative $\rho(T)$ slope. However, $\rho(T)$, does not diverge as $T \rightarrow 0$ even for $B=9 \mathrm{~T}$. 
In Fig. 1, we show representative magneto-resistance data in fields up to $B=9 \mathrm{~T}$ taken from one pixel, with the doping level $x=0.062$. In zero magnetic field, $\rho(T)$ shows a superconducting transition with $T_{c} \approx 2 \mathrm{~K}$. Magnetic field suppresses superconductivity and triggers the SIT. In the presence of the field $B=9 \mathrm{~T}$, the slope $d \rho(T) / d T$ becomes negative all the way down to $300 \mathrm{mK}$, as is typical for ordinary insulators. However, $\rho(T)$ remains relatively low even at the lowest temperature and in the highest magnetic field, although superconducting fluctuations are completely suppressed. This indicates that the 'insulating' normal state restored by the magnetic field is qualitatively different from the Mott insulator state such as observed in undoped LCO.

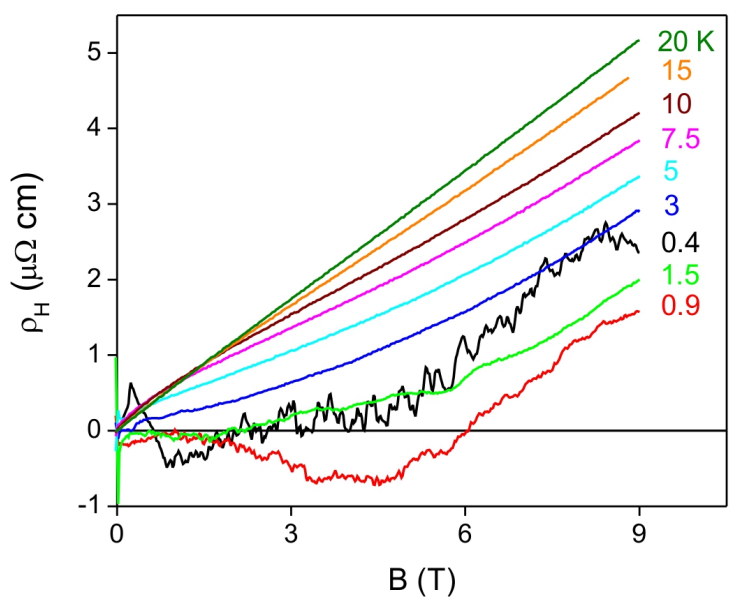

Fig. 2. The field-dependent Hall resistivity $\rho_{H}(B)$ at temperatures in the range 0.3 $\mathrm{K}<T<20 \mathrm{~K}$. For $T>1.5 \mathrm{~K}, \rho_{H}(B)$ is essentially linear except in the low-field region where superconducting fluctuations are present. Below $T_{C G}=1.5 \mathrm{~K}, \rho_{H}(B)$ shows erratic behavior characterized by jumps, spikes and kinks. The magnitude of these jumps increases when $T$ is lowered from $1.5 \mathrm{~K}$ down to $0.3 \mathrm{~K}$.

In Fig. 2, we illustrate the dependence of the Hall resistivity $\rho_{H}(B)$ on the magnetic field $B$ and temperature $T$, for the same representative doping level as in Fig. 1. For $T>T_{C G}=1.5 \mathrm{~K}$, $\rho_{H}$ is linear in $B$, as expected. However, below $T_{C G}$, as we ramp $B$ we observe dramatic jumps, spikes and kinks in $\rho_{H}(B)$. This erratic behavior is evidently not due to the instrument noise, because at $T>T_{C G}$ the noise is at least two orders of magnitude lower than the jumps at say $T$ $=0.4 \mathrm{~K}$. The magnitude of the instrument noise was verified to be independent of the sample temperature, while the amplitude of these jumps increases significantly as temperature is 
lowered below $T_{C G}$. This is additional evidence that the erratic behavior is intrinsic, and moreover that it is driven by quantum fluctuations, because thermal fluctuations diminish when $T \rightarrow 0$.

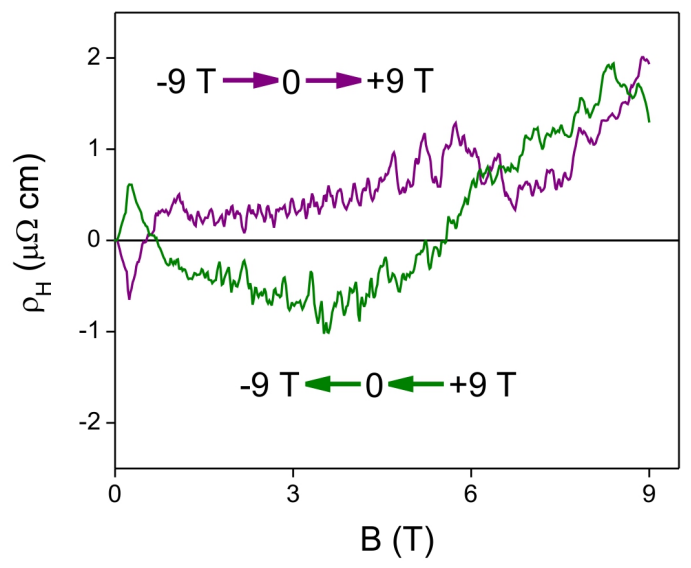

Fig. 3. Hysteresis and memory effect in $\rho_{H}(B)$ for $x=0.062$ at $T=0.4 \mathrm{~K}$. Altering the direction in which the field is ramped results in remarkable changes in $\rho_{H}(B)$.

In Fig. 3, we compare the $\rho_{H}(B)$ plot recorded from the same $x=0.062$ sample at $T=0.4 \mathrm{~K}$, while ramping the field from $B=-9 \mathrm{~T}$ to $B=9 \mathrm{~T}$, with an analogous plot recorded in an independent measurement with the field ramped in the opposite direction. The comparison shows that there is a clear hysteresis. The difference between the two $\rho_{H}(B)$ curves is so big that one even observed changes in the sign of $\rho_{H}$ at a fixed $B$.

Erratic behavior, jumps, and hysteresis can be seen in $\rho(B)$ as well, but with a much smaller relative magnitude, on the order of $0.1 \%$, in contrast to fluctuations by more than $100 \%$ in $\rho_{H}(B)$. The fact that $\rho_{H}$ is three orders of magnitude more sensitive than $\rho$ to fluctuations can be attributed to geometric factors. While $\rho_{H}$ probes only the charges across a single cross section between two parallel voltage contacts across the Hall bar width, $\rho$ is measured by averaging over a certain length $l$ (the distance between the voltage contacts along the Hall bar length) in the current direction. If $n_{l}$ is the number of charge clusters within the length $l$, this averaging reduces the effect of cluster hopping on $\rho$ by a factor of $1 / n_{l}$. 
Our linear combinatorial library contains 32 pixels of LSCO with the doping spanning the range from $x=0.062$ to $x=0.064$ and covering the SIT. We have gathering the data analogous to those shown in Figs. 1-3 for each pixel in the library, and used these to construct the $x-T-B$ phase diagram shown in Fig. 4 . In the vicinity of the doping-driven SIT, the lowtemperature ground state on the insulator side is the CCG state, so the phase competition is between the CCG and the superconducting state.

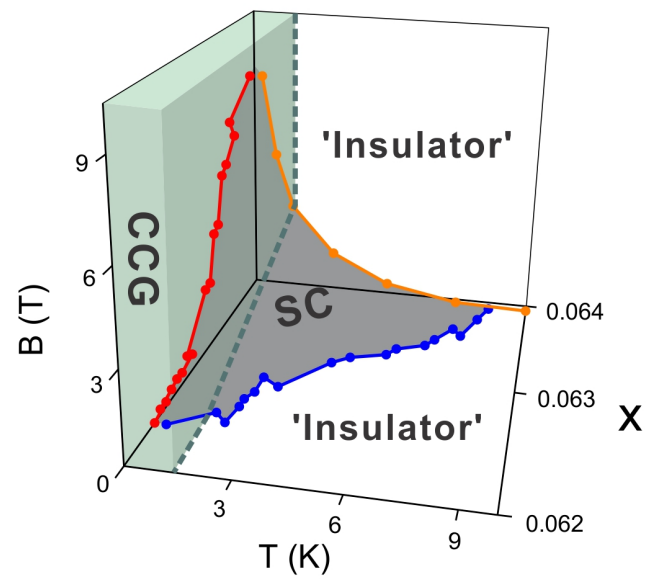

Fig. 4. The $x-T-B$ phase diagram in the vicinity of the doping-driven SIT inferred from a study of the Hall effect in the linear combinatorial LSCO library with the Sr doping level varying from $x=0.062$ to $x=0.064$ in steps $\Delta x \approx 0.00008$. As $T \rightarrow 0$, the charge-cluster glass (CCG) is competing with superconductivity for the ground state

Based on our experimental observations, we propose a unified picture to account for the anomalous electric transport in the 'insulating' state. As $T \rightarrow 0$, in the vicinity of SIT, the socalled insulator state is neither a band insulator nor a charge-transfer gap insulator, nor a Mott insulator, but rather a CCG state in which electrons are localized and clustered. The electric conductivity comes from hopping of entire clusters of charges, driven by thermal and/or quantum fluctuations. The increase of the resistivity when the temperature decreases is due to the fact that hopping gets less frequent when thermal fluctuations weaken. However, at very low temperature (and very close to the SIT), quantum fluctuations get stronger and compensate partially for the decrease of thermal fluctuations. The combined effects of thermal and quantum fluctuations make the resistivity $\rho(T)$ deviate away from the exponential $T$ dependence typically observed in band or Mott insulators ${ }^{2-4}$. 
This picture also explains in a natural way why this peculiar $\rho(T)$ dependence only occurs in the vicinity of the SIT. Envisage quantum fluctuations under discussion as quantum switching or tunneling between two microscopic states that are degenerate (or very nearly so) in energy, one describing a (micron-sized) superconducting cluster and the other a localized glassy charge cluster. In this case, frequent hopping between these two states may occur to maximize the entropy of the system. Evidently, the assumption that the superconducting and the CCG states are (nearly) degenerate in energy may be valid only in the vicinity of the doping-driven SIT; quantum fluctuations derive from the quantum critical point at SIT and decay quickly as the doping level $x$ is tuned away from $x_{c}$. Concomitantly, the $\rho(T)$ dependence approaches the standard exponential $T$ dependence as quantum fluctuations fade out when LSCO gets more and more underdoped.

The glassiness of charge can be attributed to the existence of local pinning centers such as randomly distributed $\mathrm{Sr}$ atoms, provided that the electron-lattice interaction is strong. Direct evidence that the later is indeed the case has been provided by ultrafast electron diffraction $^{15,16}$, tunneling ${ }^{17}$, and other experiments. On the other hand, the electron-phonon interaction also gives rise to the charge density wave (CDW) that has been observed in underdoped cuprates ${ }^{18-23}$. The main difference between the CDW and the CCG is that the charge modulation pattern, static or dynamic, has a well-defined period in the CDW while it is random in the CCG. However, as indicated by scanning tunneling microscopy experiments ${ }^{24}$, ${ }^{24}$, they are of the same origin: the CCG state is essentially just a glassy version of the CDW.

Acknowledgements. The experimental work was done at Brookhaven and was supported by the U.S. Department of Energy, Basic Energy Sciences, Materials Sciences and Engineering Division. I.B. was supported in part by the Gordon \& Betty Moore Foundation.

\section{References:}

1. Lee, P.A., Nagaosa, N., Wen, X.-G.: Rev. Mod. Phys. 78, 17 (2006)

2. Ando, Y., Boebinger, G.S., Passner, A., Kimura, T., Kishio, K.: Phys. Rev. Lett. 75, 4662 (1995)

3. Ando, Y., et al.: Phys. Rev. B 56, R8530 (1997)

4. Ono, S., et al.: Phys. Rev. Lett. 85, 638 (2000) 
5. Lavrov, A.N., Ando, Y., Komiya, S., Tsukada, I.: Phys. Rev. Lett. 87, 017007 (2001)

6. $\quad$ Sonier, J.E., et al.: Phys. Rev. B 76, 064522 (2007)

7. Stilp, E., et al.: Phys. Rev. B 88, 064419 (2013)

8. Wu, J., Bollinger, A.T., Sun, Y.-J., Božović, I.: Proc. Natl. Acad. Sci. (2016). doi: $10.1073 /$ pnas. 1519630113

9. Julien, M.-H., et al.: Phys. Rev. Lett. 83, 604 (1999)

10. Raičević, I., Jaroszyński, J., Popović, D., Panagopoulos, C., Sasagawa, T.: Phys. Rev. Lett. 101, 177004 (2008)

11. Shi, X., et al.: Nature Mater. 12, 47 (2013)

12. Clayhold, J.A., et al.: Rev. Sci. Instr. 79, 033908 (2008)

13. Wu, J., et al:: Nature Mater. 12, 877 (2013)

14. Wu, J., Božović, I.: APL Mater. 3, 062401 (2015)

15. Gedik, N., Yang, D.-S., Logvenov, G., Božović, I., Zewail, A.: Science 316, 425 (2007)

16. Radović, Z., Božović N., Božović I.: Phys. Rev. B 77, 092508 (2008)

17. Shim, H., Chaudhari, P., Logvenov, G., Božović I.: Phys. Rev. Letters 101, 247004 (2008)

18. Vershinin, M., et al.: Science 303, 1995 (2004)

19. da Silva Neto, E.H., et al.: Science 343, 393 (2014)

20. Chang, J., et al.: Nature Phys. 8, 871 (2012)

21. Ghiringhelli, G., et al.: Science 337, 821 (2012)

22. Le Tacon, M., et al.: Nature Phys. 10, 52 (2014)

23. Torchinsky, D. H., Mahmood, F., Bollinger, A.T., Božović I., Gedik, N.: Nature Materials 12, 387 (2013)

24. Wise, W.D., et al.: Nature Phys. 4, 696 (2008)

25. Kohsaka, Y., et al.: Science 315, 1380 (2007) 